\title{
Piece-wise Closed Functions: Corrigendum
}

\author{
J. E. Jayne and C. A. Rogers \\ Department of Mathematics, University College London WC1E 6BT, UK
}

Some of the main results of our recent paper [4] depend on Lemma 1 of that paper, and the proof of Lemma 1 depends on a result that we attributed to Hansell, [2]. Unfortunately we misquoted Hansell and stated a result that is false. We have now reinvestigated the matter in collaboration with Hansell and we hope to publish a detailed account of the necessary corrections jointly with him, [3]. In the meantime it seems appropriate to give an outline in this Journal of the necessary modifications that will be discussed in detail in the joint paper with Hansell.

The difficulty arises solely in the proof that an $\mathscr{F}_{\sigma}$ map $f$ from a metric space $X$ that is an absolute Souslin- $\mathscr{F}$ set onto a metric space $Y$ that is also an absolute Souslin-Fु set is necessarily piece-wise closed. All the results in [4], both explicit and implicit, concerning maps that are piece-wise closed, remain correct. Indeed, if Fleissner's axiom $S C \omega_{2}$ holds, see [1], then all the results in [4] also hold. Further, the result italicized above holds, without appeal to any special axioms, if we make any one of the following additional assumptions about $f$.

(a) Each point of $X$ has a neighbourhood that is mapped by fonto a set in $Y$ that is $\sigma$-locally of weight at most $\aleph_{1}$.

(b) $f^{-1}(y)$ is compact for each $y$ in $Y$.

(c) $f$ is an open map.

By introducing any one of these additional assumptions in appropriate ways, all the results in $[4]$ on $\mathscr{F}_{\sigma}$ maps may be corrected.

\section{References}

1. Fleissner, W.G.: An axiom for nonseparable Borel theory. Trans. Amer. Math. Soc. 251, 309-328 (1979)

2. Hansell, R.W.: Borel measurable mappings for nonseparable metric spaces. Trans. Amer. Math. Soc. 161, 145-169 (1971)

3. Hansell, R.W., Jayne, J.E., Rogers, C.A.: Piece-wise closed functions and almost discretely $\sigma$-decomposable families. To appear

4. Jayne, J.E., Rogers, C.A.: Piece-wise closed functions. Math. Ann. 255, 499-518 (1981) 